\title{
Feline Aortenthrombose - Ist eine Prophylaxe sinnvoll?
}

\author{
Alan Kovacevic
}

Katzen, die unter einer Kardiomyopathie leiden, haben ein hohes Risiko für eine feline Aortenthrombose. Die optimale Therapie der Aortenthrombose variiert dabei in Abhängigkeit vom individuellen Zustand des Patienten. Die Prognose betroffener Tiere ist ungünstig. Katzen, die mit Clopidogrel behandelt wurden, zeigen jedoch eine deutlich längere Überlebenszeit als Katzen, die Acetylsalicylsäure erhielten.

\section{Einleitung}

Die feline Aortenthrombose ist die häufigste Form der Arterienthrombose. Sie kennzeichnet sich durch eine ischämische Myo-/Neuropathie der Hintergliedmaßen. Dabei kommt es zu einem akuten Verschluss der Aorta durch einen verschleppten Thrombus. Dieser ist meist im Bereich der Aufzweigung der abdominalen Aorta (Trifurcatio aortae) lokalisiert und wird auch als Sattelthrombus bezeichnet.

Im Patientengut einer Überweisungsklinik liegt die Prävalenz der felinen Aortenthrombose im Allgemeinen bei ca. $0,5 \%$. In der Regel handelt es sich um die Komplikation einer Kardiomyopathie. So wird davon ausgegangen, dass $12-41 \%$ der Katzen mit einer hypertrophen Kardiomyopathie ( $\boldsymbol{A}$ bb.1) von einer Aortenthrombose betroffen sind $[6,8]$. Nur selten sind andere Auslöser der Thrombose zu erkennen, beispielsweise:

- Entzündungen

- Infektionen

- Neoplasien

- Hyperthyreose

Nur das gleichzeitige Vorhandensein mehrerer Faktoren, die bei Katzen mit einer Kardiomyopathie oftmals vorhanden sind, kann eine Thrombose auslösen.
Diese Faktoren werden als Virchow'sche Triade bezeichnet. Dazu zählen:

- Verlangsamung im Blutfluss (Stau)

- Zustand der Hyperkoagulabilität

- Schädigung des Endothels

\section{Symptomatik}

Das klinische Bild der felinen Aortenthrombose kennzeichnet sich durch:

- perakut auftretende Schmerzen

- Parese oder Paralyse der Hintergliedmaßen

- kalte, zyanotische oder zyanotischgraue Ballen

- Durchblutungsstörungen des Krallenbetts (keine/minimale Blutung nach anschneiden, abnorm dunkles, schwarzes Blut)

- harte, kalte Muskulatur der Hinterbeine

- kaum oder nicht palpierbarer Puls der A. femoralis

Ist der Thrombus weiter kranial lokalisiert, sind zudem folgende Symptome möglich:

- Parese oder Paralyse der Vordergliedmaßen

- Urämie (Thrombose der Nierenarterien)

- Durchfall (Thrombose der Mesenterialarterien)

\section{Diagnose}

Der klinische Befund ist oft zur Diagnosestellung ausreichend. Labordiagnostisch fällt auf, dass die Muskelenzyme im Blutserum (AST, CK) einige Stunden nach Eintreten der felinen Aortenthrombose um das Vielfache über den Normwert ansteigen.

Die Diagnose kann durch eine Strömungsmessung am Hinterbein mittels Dopplersonografie ( $\triangleright$ Abb.2), eine Ultra- schalluntersuchung der abdominalen Aorta sowie eine CT-Untersuchung ( $\triangleright$ Abb.3) des Abdomens gesichert werden.

\section{Therapie}

Der Schwerpunkt der Therapie besteht in einer guten Analgesie.

Dazu eignet sich die Applikation von Buprenorphin, Butorphanol oder Fentanyl ( $\downarrow$ Tab.1). Bei Unruhe und Angstzuständen ist zudem eine Sedation mittels Acepromazin empfehlenswert.

Das primäre Herzproblem ist zudem mit einem ACE-Hemmer sowie einem $\mathrm{Ca}^{++}$Kanal-Blocker zu therapieren. Liegt ein kongestives Herzversagen vor, sollte zusätzlich Furosemid bis zur Besserung der Atmung verabreicht werden. Es sollten 1-2 $\mathrm{mg} / \mathrm{kg}$ als Bolus (nach Bedarf alle 30-120 Minuten wiederholen) oder bei refraktärer Dyspnoe 0,25-0,5 mg/kg/h

Tab. 1 Therapie der felinen Aortenthrombose.

\begin{tabular}{|c|c|}
\hline Medikament & Dosierung \\
\hline \multicolumn{2}{|l|}{ Analgetika } \\
\hline Buprenorphin & $\begin{array}{l}0,01-0,03 \mathrm{mg} / \mathrm{kg} \text { s.c. } \\
\text { oder i. m. alle } 4-6 \mathrm{~h}\end{array}$ \\
\hline Butorphanol & $\begin{array}{l}0,2-0,4 \mathrm{mg} / \mathrm{kg} \text { i. v. } \\
\text { oder i. m. nach Bedarf }\end{array}$ \\
\hline Fentanyl & $\begin{array}{l}5 \mu \mathrm{g} / \mathrm{kg} \text { als Bolus i. v. } \\
5 \mu \mathrm{g} / \mathrm{kg} / \mathrm{h} \text { als } \\
\text { Dauertropf i.v. }\end{array}$ \\
\hline \multicolumn{2}{|l|}{ Sedativum } \\
\hline Acepromazin & $\begin{array}{l}0,01-0,05 \mathrm{mg} / \mathrm{kg} \text { i.m. } \\
\text { oder i.v. }\end{array}$ \\
\hline \multicolumn{2}{|l|}{ Antikoagulans } \\
\hline Heparin & $\begin{array}{l}100-300 \mathrm{IE} / \mathrm{kg} \text { s.c., } \\
\text { gefolgt von } \\
50-100 \mathrm{IE} / \mathrm{kg} \text { alle } 8 \mathrm{~h}\end{array}$ \\
\hline
\end{tabular}


als Dauertropf verabreicht werden. Ein Sauerstoffkäfig ist zur Optimierung der Oxygenierung vorteilhaft. Die Gabe von $\beta$-Blockern ist im kongestiven Zustand kontraindiziert.

Eine aktive Thrombolyse mittels gewebespezifischem Plasminogenaktivator (t-PA) oder Streptokinase ist nicht ratsam. Studien konnten nachweisen, dass die Letalitätsrate nach der aktiven Thrombolyse sehr hoch (etwa 50\%) und somit kontraproduktiv ist. Man geht davon aus, dass die aktive Thrombolyse zu relevanten Reperfusionsstörungen führt und den Patienten daher destabilisiert.

Zudem wurde eine aktive mechanische Thrombolyse mithilfe spezieller Katheter bei Katzen beschrieben [7]. Dabei wird ein Katheter bis zum Thrombus vorgeführt und dieser durch Spülung und $\mathrm{Ab}$ saugen entfernt. In einer Studie wurden 6 Katzen auf die beschriebene Weise therapiert. Bei 5 Katzen konnte der Thrombus entfernt werden. Jedoch konnten nur 3 der 6 Katzen aus der Klinik entlassen werden, die anderen starben an den Folgen des Eingriffs. Die aktive mechanische Thrombolyse ist daher nicht empfehlenswert.

Das Wachstum des Thrombus sollte aber mithilfe von Heparin verhindert werden. Dazu liegen diverse Heparin-Protokolle vor. Es empfiehlt sich, initial 100-300 IE/ $\mathrm{kg}$ Heparin zu verabreichen, gefolgt von 50-100 IE/kg alle 8 Stunden.

Es ist darauf zu achten, dass das Heparin nach 3-4 Tagen langsam ausgeschlichen wird.

Ein zu rascher Abbruch kann zu einer erhöhten Gerinnungstendenz führen.

Dalteparin (100-150 IE/kg alle 8-12 Stunden) und Enoxaparin (1 mg/kg alle 8-12 Stunden) erwiesen sich im Vergleich zu Heparin nicht als vorteilhaft. Die deutlich höheren Kosten von fraktioniertem Heparin sind somit nicht sinnvoll. Auch eine Antikoagulation mittels Warfarin ist aufgrund der übermäßigen und schwer kontrollierbaren Gerin- nungshemmung nicht empfehlenswert. Heparin und fraktioniertes Heparin führen nicht zur bedeutenden Thrombolyse. Die Verabreichung dieser Mittel ist als prophylaktische Maßnahme in der akuten Phase der Erkrankung zu verstehen, die das weitere Wachstum des Thrombus verhindert.
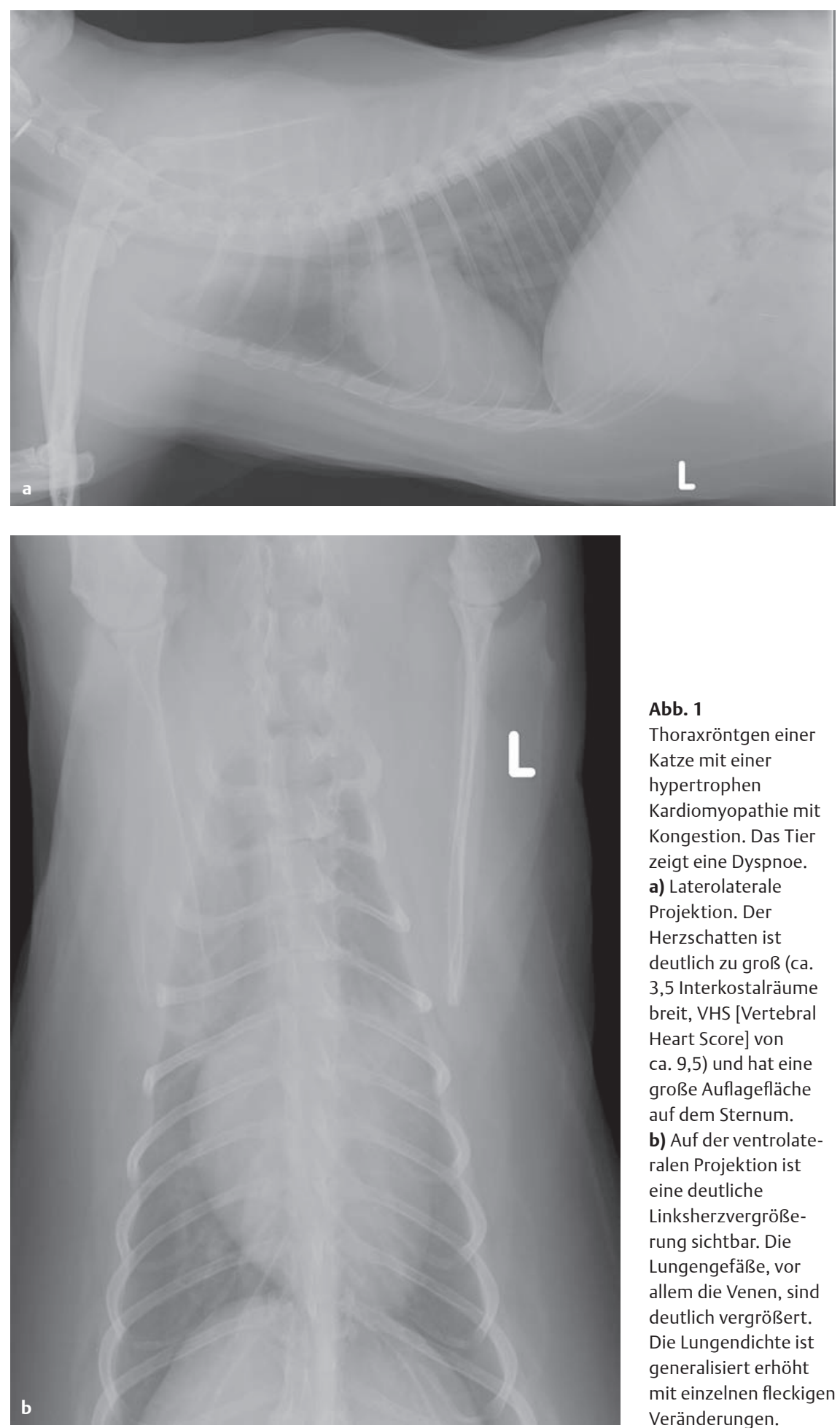

Abb. 1

Thoraxröntgen einer Katze mit einer hypertrophen Kardiomyopathie mit Kongestion. Das Tier zeigt eine Dyspnoe. a) Laterolaterale Projektion. Der Herzschatten ist deutlich zu groß (ca. 3,5 Interkostalräume breit, VHS [Vertebral Heart Score] von ca. 9,5) und hat eine große Auflagefläche auf dem Sternum. b) Auf der ventrolateralen Projektion ist eine deutliche Linksherzvergrößerung sichtbar. Die Lungengefäße, vor allem die Venen, sind deutlich vergrößert. Die Lungendichte ist generalisiert erhöht mit einzelnen fleckigen Veränderungen. 


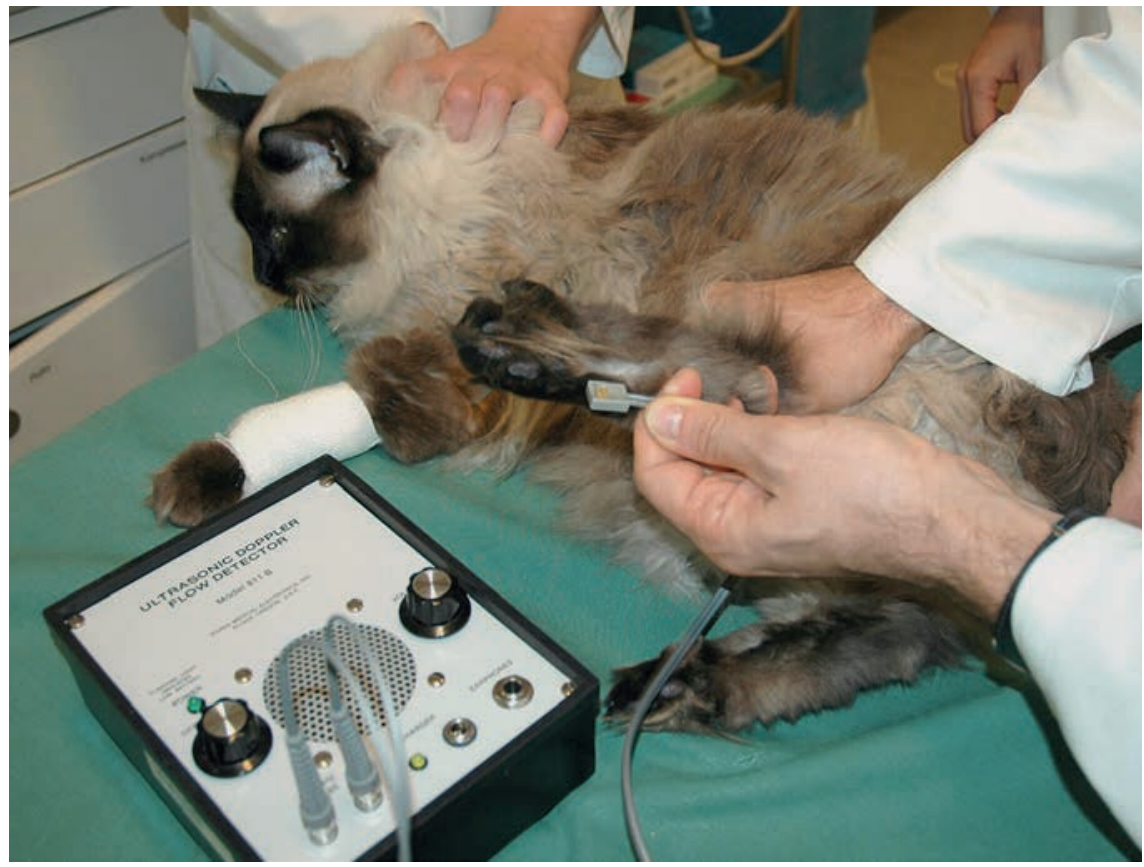

Abb. 2 Mittels Ultraschall Doppler kann an der untersuchten Pfote festgestellt werden, ob es eine Blutströmung gibt oder diese durch einen Verschluss des Gefäßes nicht nachweisbar ist.

\section{Prophylaxe}

Prophylaktische Maßnahmen werden bei herzkranken Katzen diskutiert, um einer Thrombose vorzubeugen.

\section{Nach dem Ausschleichen des Heparins sollte eine lebenslange Thrombose- Prophylaxe durchgeführt werden.}

Es liegen jedoch keine Studien vor, die ein erfolgreiches Prophylaxe-Protokoll der felinen Aortenthrombose belegen. Zudem ist noch ungeklärt, bei welcher Herzerkrankung und in welchem Krankheitsstadium die antithrombotische Therapie eingeleitet werden sollte. Die Erweiterung des linken Vorhofs (LA/Ao $>1,6-1,8)$ wird in der Fachliteratur bereits als Indikation diskutiert.

Als Mittel der Wahl stehen dabei Acetylsalicylsäure (ASS) und Clopidogrel zur Verfügung ( $\triangleright$ Tab.2).

Tab. 2 Prophylaxe-Protokolle.

\begin{tabular}{|l|l|}
\hline Medikament & Dosierung \\
\hline ASS & $\begin{array}{l}5 \mathrm{mg} / \text { Katze } \mathrm{p} . \mathrm{o} . \\
\text { alle } 3 \text { Tage }\end{array}$ \\
\hline Clopidogrel & $\begin{array}{l}18,75 \mathrm{mg} / \text { Katze } \mathrm{p} . \mathrm{o} . \\
\text { täglich }\end{array}$ \\
\hline
\end{tabular}

\section{ASS}

- führt zur irreversiblen Acetylierung der Cyclooxygenase und

- blockiert somit die Bildung von Thromboxan.

Für ASS liegen 2 Protokolle mit unterschiedlichen Dosierungen vor. Dabei Protokoll mit der höheren Dosis $(81 \mathrm{mg} /$ Katze alle 3 Tage) keine bessere Hemmung der Koagulation erzeugt, aber zu mehr Nebeneffekten führt. Empfehlenswert ist daher das sogenannte „lowdose“-Protokoll, bei dem alle 72 Stunden $5 \mathrm{mg} /$ Katze verabreicht werden [10].

\section{Clopidogrel}

- führt zur Inhibition der ADP-Rezeptoren an den Thrombozyten,

- reduziert die Bindung von Fibrinogen an den Von-Willebrand-Faktor und

- provoziert die Inhibition der GP-IIb/IIIa-Rezeptoren.

Seit Kurzem liegen erste Resultate einer multizentrischen, randomisierten, verblindeten und prospektiven Studie zur Rezidivprophylaxe der felinen Aortenthrombose vor [3]. In die Studie wurden Katzen aufgenommen, die vor 1-3 Monaten an einer Aortenthrombose erkrankkonnte nachgewiesen werden, dass das

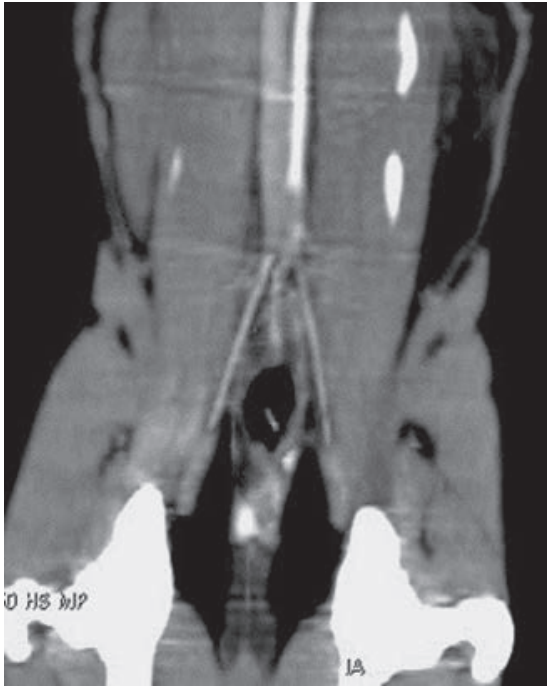

Abb. 3 CT-Aufnahme mit Kontrast. Der Kontrastmittelfluss wird in der Abdominalaorta auf der Höhe der Trifurkation geblockt.

ten. Die Studienpopulation (72 Katzen) wurde in 2 Gruppen aufgeteilt: Die 1. Gruppe erhielt ASS (81 mg p.o. alle 3 Tage), die 2. Gruppe hingegen Clopidogrel (18,75 mg p.o. $1 \times$ täglich). Die Katzen der Clopidogrel-Gruppe zeigten eine mediane Überlebenszeit von 443 Tagen und die der ASS-Gruppe von 192 Tagen. Der Vorteil von Clopidogrel war somit signifikant ( $p=0,019)$. Da die Katzen dieser Studie jedoch bereits an einer Thrombose erkrankt waren, kann das Resultat nicht ohne Weiteres auf alle herzkranken Katzen in unterschiedlichen Krankheitsstadien übertragen werden.

\section{Prognose}

Die meisten Katzen mit einer felinen Aortenthrombose sterben an den Folgen der primären Herzerkrankung. Die Überlebenszeit beträgt 51-443 Tage [1,3,5,9]. Eine signifikant schlechtere Prognose zeigen Katzen mit:

- Untertemperatur

- mehreren befallenen Gliedmaßen

- erhöhtem Phosphat-Serumspiegel 


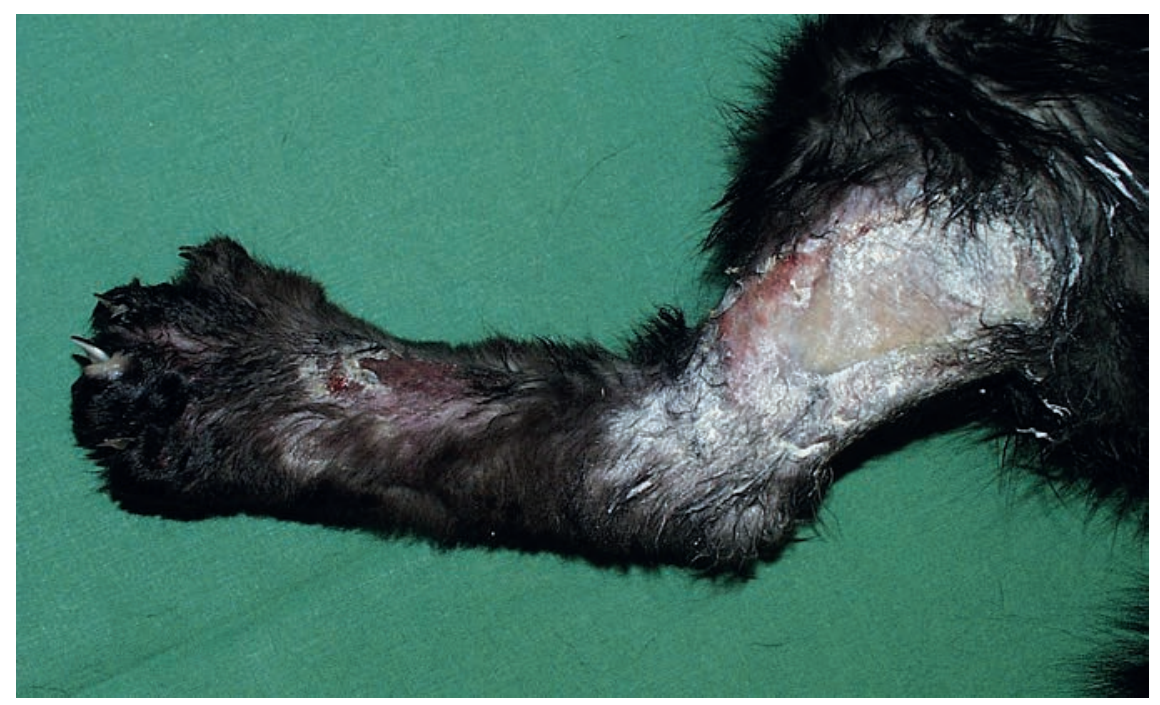

Abb. 4 Katze mit Gangrän.

Weitere negative prognostische Faktoren sind:

- kongestives Herzversagen

- Arrhythmien

- disseminierte intravasale Koagulopathien

- nicht responsive Hypothermie

- Hyperkaliämie

- Thromben im linken Atrium/Ventrikel

- Multiorganversagen

- neurologische Defizite

- blutiger Durchfall

- akutes Nierenversagen

Eine dezente Rückkehr der motorischen Funktionen zeigt sich bei den meisten Katzen nach 3-4 Tagen. Mit einer teilweisen oder vollständigen Wiederherstellung der motorischen Funktion der Hinterbeine ist innerhalb von 1-6 Wochen zu rechnen. Bei 5\% der Tiere kommt es nach einigen Tagen zu Komplikationen, beispielsweise einer Nekrose und einem Gangrän ( Abb.4). In solchen Fällen sind eine Amputation der Gliedmaße oder eine Euthanasie des Tieres indiziert.

\section{.konkret}

Das Rezidivrisiko einer Thrombose beträgt $24-75 \%$, sodass eine lebenslange Prophylaxe unbedingt empfehlenswert ist $[2,4,5]$.

\section{Fazit}

Um das Rezidivrisiko der Aortenthrombose zu senken, sollte eine lebenslange Prophylaxe durchgeführt werden. Aufgrund der längeren medianen Überlebenszeit empfiehlt sich dabei die Applikation von Clopidogrel. Sollte ASS beispielsweise aus Kostengründen oder aufgrund seines 3-tägigen Applikationsintervalls als Antikoagulans verabreicht werden, ist das „low-dose“-Protokoll ( $5 \mathrm{mg} /$ Katze alle 3 Tage) zu bevorzugen. Unklar bleibt aber weiterhin, bei welcher Herzerkrankung und in welchem Krankheitsstadium eine Indikation zur Prophylaxe besteht.

\section{Online zu finden unter}

http://dx.doi.org/10.1055/s-0033-1361537

\section{Literatur}

1 Atkins CE, Gallo AM, Kurzman ID et al. Risk factors, clinical signs, and survival in cats with a clinical diagnosis of idiopathic hypertrophic cardiomyopathy: 74 cases (1985-1989). J Am Vet Med Assoc 1992; 201 (4): 613-618

2 Fox PR. Evidence for or against efficacy of beta-blockers and aspirin for management of feline cardiomyopathies. Vet Clin North Am Small Anim Pract 1991; 21 (5): 1011-1022

3 Hogan D, Fox P, Jacob K et al. Analysis of the feline arterial thromboembolism: clopidogrel vs. aspirin trial (FAT cat). Proceedings ACVIM 2013; 177 
4 Laste NJ, Harpster NK. A retrospective study of 100 cases of feline distal aortic thromboembolism: 1977-1993. J Am Anim Hosp Assoc 1995; 31 (6): 492-500

5 Moore KE, Morris N, Dhupa N et al. Retrospective study of streptokinase administration in 46 cats with arterial thromboembolism. J Vet Emerg Crit Care 2000; 10: 245-257

6 Peterson EN, Moise NS, Brown CA et al. Heterogeneity of hypertrophy in feline hypertrophic heart disease. J Vet Intern Med 1993; 7 (3): 183-189
7 Reimer SB, Kittleson MD, Kyles AE. Use of Rheolytic thrombectomy in the treatment of feline distal aortic thromboembolism. J Vet Intern Med 2006; 20 (2): 290-296

8 Rush JE, Freeman LM, Fenollosa NK et al. Population and survival characteristics of cats with hypertrophic cardiomyopathy: 260 cases (1990-1999). J Am Vet Med Assoc 2002; 220 (2): 202-207

9 Schoeman JP. Feline distal aortic thromboembolism: A review of 44 cases (1990-1998). J Feline Med Surg 1999; 1 (4): 221-231
10 Smith SA, Tobias AH, Jacob KA et al. Arterial thromboembolism in cats: acute crisis in 127 cases (1992-2001) and long-term management with low-dose aspirin in 24 cases. J Vet Intern Med 2003; 17 (19): 73-83

\section{Dr. med. vet. Alan Kovacevic}

DECVIM-CA/cardiology

Vetsuisse-Fakultät Universität Bern

Departement für klinische Veterinärmedizin

Abteilung Kardiologie

Längass-Straße 128

$\mathrm{CH}-3012$ Bern

alan.kovacevic@vetsuisse.unibe.ch 